Colleagues in Oncology have suggested several protocols to disclose and discuss the details of the clinical condition of their patients. Some of these protocols appear to be suitable to be adopted for use in counselling parents.

This paper will advocate the adoption of the SPIKES protocol (with permission) that was originally described to disclose unfavourable clinical information to patients with cancer.

The six steps of Modified SPIKES:

$\mathrm{S}$-Setting up the interview

$\mathrm{P}$-Assessing the parents' Perception

I-Obtaining the parents' Invitation

$\mathrm{K}$-Giving Knowledge and information

E-Addressing the parents' Emotions with empathic responses

S-Strategy and Summary

Clips demonstrating the above steps of counselling will be shown. The video was recorded with real parents of a premature baby.

Conclusion A structured standard approach will help the healthcare professional to perform such a stressful task efficiently in a reproducible model. This may serve as a training tool too. To my knowledge, the counselling video of this presentation is the first of its kind in utilising a structured approach with the participation of real parents.

\section{G454(P) NEONATAL AMOEBIASIS MAY NOT BE AS RARE AS WE THOUGHT}

ZG Abusalah. Neonatal Intensive Care Unit, Mediclinic City Hospital, Dubai, UAE

\subsection{6/archdischild-2020-rcpch.392}

Introduction Amoebiasis is a parasitic infection caused by a protozoan called Entamoeba Histolytica (EH). It is responsible for up to 100,000 deaths every year worldwide. Only a handful of cases of neonatal and infantile amoebiasis has been reported.

\section{Aims}

- To highlight the incidence of this infection in the neonatal age group.

- To demonstrate the use of rapid antigen detection as a tool for diagnosis in areas with limited resources.

Methods A total of six babies presented to the Neonatal Intensive Care Unit (NICU) over a period of 24 months. All of them but one shared a universal symptom of passing stool mixed with fresh blood.

The first baby was born at 25 weeks of gestation. At the age of 2 weeks, he developed bloody stool associated with abdominal distension. He could not maintain adequate spontaneous breathing effort. Therefore, he had to be ventilated.

Other two babies were preterm babies born at 26 and 25 weeks. However, they developed the infection at a corrected gestation of 38 and 44 weeks respectively. They remained well with no other associated symptoms.

The other two babies were term well babies. They were reported to pass fresh blood mixed with their stool shortly after birth while on the postnatal ward. Interestingly, with one of these two babies, there was a history of confirmed neonatal amoebiasis with his older sibling. The sibling was born two years ago in a different country.

The last baby was born at 23 weeks and 6 days of gestation. He developed small bowel perforation as a complication of Necrotising Enterocolitis. His infection has manifested by sudden increase in his ileostomy output associated with raised C-reactive protein.

The diagnosis of amoebiasis was confirmed by the detection of $\mathrm{EH}$ antigens in stool (chromatographic immunoassay) in all babies.

Conclusion Neonatal amoebiasis appears to be commoner than previously reported. In areas with high prevalence, It should be suspected in babies presenting with passing fresh blood in their stool.

The diagnosis may be reliably made using rapid $\mathrm{EH}$ antigen detecting test.

\section{G455(P) ABSTRACT WITHDRAWN}

\section{G456(P) ABSTRACT WITHDRAWN}

\section{G457(P) ADMISSION PROCESSES IN HPA-AN GENERAL HOSPITAL, MYANMAR}

${ }^{1}$ E Ivey, ${ }^{2} \mathrm{HH}$ Win, ${ }^{2} \mathrm{HMM}$ Chan, ${ }^{2} \mathrm{TT}$ Oo, ${ }^{1} \mathrm{M}$ Wootton, ${ }^{3} \mathrm{I}$ Maconochie. ${ }^{1} \mathrm{Global}$ Child Health, Royal College of Paediatrics and Child Health, London, UK; ${ }^{2}$ Child Ward, Hpa An General Hospital, Hpa An, Myanmar; ${ }^{3}$ Paediatric Emergency Medicine, Imperial College Healthcare Trust, London, UK

\subsection{6/archdischild-2020-rcpch.393}

Background Myanmar faces challenges of rising patient numbers, with state and district hospitals particularly feeling the strain. In Kayin state $78 \%$ of the population live in rural areas. Affected by recent conflict and economic migration of the working-age population, children under 15 make up $36 \%$ of the population. The hospital presented here is a 200-bedded state hospital, serving a population close to 800,000 . There is little data describing patterns in acute paediatric admissions to state hospitals in Myanmar.

Aim To describe the processes of paediatric admission to state hospital and the characteristics of admitted children by age, referral source and rates of admissions.

Methods Logbook records from the emergency department (OPD) and Child Ward (CW) were reviewed for the period March 2018-February 2019. OPD attendances were analysed by age and outcome. Records were reviewed to establish the proportion of admitted patients referred from other hospitals.

Results Potential outcomes from OPD assessment were discharge, daily OPD review, clinic referral or admission. Children $<12$ y with medical problems are admitted to CW; children $>12 \mathrm{y}$ and those $<12 \mathrm{y}$ with surgical problems are admitted to adult wards, under adult teams.

Children accounted for $27 \%$ of OPD attendances. On average, 1475 children attended OPD each month. 40.3\% of children attending OPD were admitted to $\mathrm{CW}$ on average, rising significantly to $48.8 \%(\mathrm{p} \leq 0.001)$ in the rainy season (June to September). Admissions were highest in children $<2$ years. Referrals from other hospitals represented $4.7 \%$ of admissions and this figure rose throughout the year. 